\title{
A influência da saúde bucal no controle glicêmico, sob orientação interdisciplinar
}

\author{
The influence of oral health on glycemic control, \\ under interdisciplinary guidance
}

\author{
Isabella de Oliveira Bilitardo ${ }^{1}$, Beatriz Nogueira Leite ${ }^{2}$, Tatiana Ribeiro de Campos Mello
}

Bilitardo IO, Leite BN, Mello TRC. A influência da saúde bucal no controle glicêmico, sob orientação interdisciplinar / The influence of oral health on glycemic control, under interdisciplinar guidance. Rev Med (São Paulo). 2020 maio-jun.;99(3):258-65.

\begin{abstract}
RESUMO: O controle glicêmico é essencial não só para o paciente diabético, mas também para qualquer outro paciente que zele pelo seu bem-estar geral. Saber que uma boa saúde bucal interfere positivamente nesse controle favorece o paciente, desde que ele adquira esse conhecimento durante seus atendimentos médicos e odontológicos. Esse estudo é do tipo quantitativo transversal e por meio de um questionário avaliou-se o conhecimento de 196 pacientes do XVI Projeto Antonio Prudente (Feira de Saúde 2016) sobre a influência da saúde bucal no controle glicêmico, além de verificar a orientação médica e odontológica aos pacientes sobre essa influência e relacionar o tipo de orientação profissional com a glicemia deles. Poucos entrevistados consideraram a saúde bucal importante para controlar a glicemia, pelo fato de que a minoria dos pacientes é orientada em conjunto pelos médicos e dentistas sobre a necessidade do cuidado da saúde como um todo. Concluiu-se que é extremamente importante ter serviços de saúde públicos e privados multidisciplinares aos diabéticos para educá-los no autocuidado, gerar melhor comunicação entre os profissionais e garantir melhor qualidade de vida aos pacientes após controle eficaz da glicemia capilar.
\end{abstract}

Descritores: Glicemia, Saúde bucal, Equipe de assistência ao paciente.

\begin{abstract}
Glycemic control is essential not only for the diabetic patient, but also for any other patient who watches over their general well-being. Therefore, knowing that good oral health interferes positively in this control favors the patient, if he acquires this knowledge during his medical and dental appointments. This is a cross-sectional quantitative study which through a questionnaire aimed to evaluate the knowledge of 196 patients of the XVI Projeto Antonio Prudente (Health Fair 2016) on the influence of oral health on glycemic control, as well as to verify the medical and dental orientation to patients about this influence and to relate the type of professional orientation with their blood glucose. Few interviewees considered oral health important for glycemic control because the minority of patients is jointly advised by doctors and dentists about the need for multidisciplinary diabetic care to better control the disease. It was concluded that it is extremely important to have multidisciplinary public and private health services for diabetics to educate them in self-care, to generate better communication among professionals and to guarantee better quality of life for patients after effective control of capillary glycemia.
\end{abstract}

Keywords: Blood glucose; Oral health; Patient care team.

\footnotetext{
1. Discente de Medicina na Universidade de Mogi das Cruzes, Mogi das Cruzes, SP, BR. https://orcid.org/0000-0002-3325-0212.Email: isabellabilitardo@ hotmail.com.

2. Discente de Medicina, Universidade de Mogi das Cruzes, Mogi das Cruzes, SP. https://orcid.org/0000-0001-8570-5306. Email: bnleite@hotmail.com 3. Graduação em Odontologia na Universidade de Mogi das Cruzes. Docente na Universidade de Mogi das Cruzes. Mestrado em Deontologia e Odontologia Legal pela Universidade de São Paulo. Doutorado em Saúde Pública pela Universidade de São Paulo. Pós-Doutorado pela Universidade de Campinas. https://orcid.org/0000-0003-4966-3633. Email: tatianar@umc.br.

Endereço para correspondência: Isabella de Oliveira Bilitardo. Av. João XXIII, nº 3904, Reserva Granja Anita (Quadra G e Lote 29), Cesar de Souza. Mogi das Cruzes, SP, Brasil. CEP 08830-000.
} 


\section{INTRODUÇÃO} Diabetes Mellitus é uma doença
crônica multifatorial caracterizada por distúrbios metabólicos em decorrência da exposição à hiperglicemia, pois existe uma resistência à insulina endógena ou produção insuficiente dela ${ }^{1}$. Segundo dados da Federação Internacional de Diabetes (IDF) em 2014, um contingente de 387 milhões de pessoas no mundo apresentam a doença, uma prevalência global de $8,3 \%$, e com expectativa de aumento de $53 \%$ até 2035 . Desse total, estima-se que 11,9 milhões sejam brasileiros e que, em 2035, 19,2 milhões de pessoas no Brasil terão a doença ${ }^{2}$.

Para se evitar as complicações da exposição à hiperglicemia, o paciente precisa ser orientado quanto às mudanças de estilo de vida e os fatores que interferem no controle da doença. Sem essas informações o paciente não consegue entender a importância do tratamento correto e a adesão a ele se torna menor. Para uma melhora do paciente com diabetes devem ser consideradas as condições socioeconômicas e aspectos culturais, e não só o indivíduo e a família isoladamente. Outro ponto importante é o tratamento multidisciplinar, ou seja, se atentar a detalhes que não são da área médica, mas que poderiam resolver uma hiperglicemia persistente.

Dentre as complicações do diabetes a doença periodontal está entre as seis mais prevalente e é considerada relevante por ter aumento na sua frequência e progressão ${ }^{3}$. E, de modo paralelo, das associações observadas entre condição de saúde bucal e as patologias sistêmicas crônicas e multifatoriais, a doença periodontal é a que faz maior ligação com Diabetes Mellitus ${ }^{4}$. Não se pode esquecer, então, que não é apenas diabetes que está relacionada com doenças odontológicas, pois o processo inflamatório e infeccioso, predominantemente anaeróbias gram-negativas, também se relaciona com outros acometimentos sistêmicos como acidente vascular cerebral, infecções pulmonares, distúrbios gastrointestinais e nascimento de bebes de baixo peso e/ou prematuros ${ }^{5}$.

O tratamento da periodontite em pacientes diabéticos interfere positivamente nos marcadores da doença, tais como redução do índice glicêmico, citocinas pró-inflamatórias, metaloproteinases da matriz e proteína $\mathrm{C}$ reativa ${ }^{6}$. É bem documentado que infecções agudas e condições inflamatórias aumentam os níveis séricos de glicose e a utilização de insulina e, por conseguinte, complicam o controle metabólico da diabetes. Deve-se considerar, então, a atenção ao tratamento dentário como coadjuvante ao tratamento doenças sistêmicas, uma vez que, como já comentado, a infecção bucal crônica está relacionada a diversas outras patologias, podendo alterar o curso de controle e seguimento delas ${ }^{7}$. Quando foi tratada a condição periodontal de pacientes em hemodiálise, houve melhora significativa também em alguns dos índices inflamatórios como a creatina, em hemoglobina e hematócrito quando comparados o antes e após tratamento ${ }^{8}$.

Uma meta-análise mostrou que o tratamento periodontal levou a uma redução da HbA1c, hemoglobina glicosada, de cerca de $0,4 \%$. Isso pode parecer pouco expressivo, mas os autores dessa revisão salientam que, até o momento, mesmo uma ligeira redução da HbA1c pode diminuir o risco de complicações microvasculares. Por conseguinte, uma redução desta magnitude pode levar a uma redução na mortalidade ao nível da população de mais do que $10 \%$.

Sabe-se que a baixa aderência ao tratamento e a displicência em relação às mudanças de hábitos fazem com que cerca de $50 \%$ dos indivíduos que são portadores de doenças crônicas, como o diabetes, não evoluam bem no contexto da doença ${ }^{10}$. Pode-se dizer que isso se deve pelo fato de o paciente não entender, muitas vezes, a importância do tratamento correto, as consequências sérias que a negligência leva e os fatores que impedem a melhora de sua saúde. O médico, conhecendo melhor todos os aspectos que interferem positiva e negativamente na taxa glicêmica, como por exemplo, a doença periodontal, consegue orientar melhor e contribuir para o bem-estar do paciente 9 .

Sendo assim, tem-se como objetivo desta pesquisa analisar o conhecimento de 196 pacientes, participantes de uma feira de saúde universitária, sobre a influência da saúde bucal no controle glicêmico. Além disso, procurouse avaliar se tanto médicos quanto dentistas orientam os pacientes sobre essa questão.

\section{MATERIAL E MÉTODOS}

O estudo foi realizado na cidade de Mogi das Cruzes, localizada na região do Alto Tietê do estado de São Paulo, fica a aproximadamente 50 quilômetros da cidade de São Paulo e possui população estimada em 433.901 habitantes ${ }^{11}$ (Instituto Brasileiro de Geografia e Estatística, 2017). A rede de atenção primária de saúde do município possui vinte e uma Unidades Básicas de Sáude (UBS) e doze Unidades de Saúde da Família (USF). Vinte UBSs e cinco USFs contam com atendimento odontológico.

Trata-se de um estudo quantitativo transversal, realizado no XVI Projeto Antonio Prudente - Feira de Saúde do Centro Acadêmico Antonio Prudente (C.A.A.P.), que é um órgão suplementar do curso de Medicina da Universidade de Mogi das Cruzes. Esse projeto anual é organizado pelos alunos da gestão do C.A.A.P. e conta com cerca de 350 alunos e profissionais da saúde voluntários que realizam uma média de 2000 atendimentos, sejam eles médico, psicológico, nutricional, esportivo, biomédico ou odontológico.

A amostra do estudo constitui-se de 196 pacientes do XVI Projeto Antonio Prudente que atenderam aos seguintes critérios de inclusão pré-estabelecidos: ter mais de 18 anos e ter realizado o teste de glicemia capilar no setor de triagem da feira de saúde. Os dados foram obtidos por meio de uma 
abordagem ao paciente seguida de uma breve explicação da proposta dos pesquisadores para permitir que o entrevistado tivesse a liberdade e a espontaneidade necessárias para o enriquecimento da investigação. As entrevistas foram realizadas nas salas de espera dos consultórios durante os dois dias da feira de saúde.

$\mathrm{O}$ instrumento de investigação foi um questionário composto por dados de identificação dos sujeitos e questões norteadoras referentes ao fornecimento de informações sobre a influência da saúde bucal no controle glicêmico por médicos e por dentista aos pacientes. No questionário aplicado, havia também perguntas para avaliar se o paciente tinha sangramento gengival enquanto utilizava fio dental, se ele era avaliado por médicos e dentistas com frequência, se ele considerava sua saúde bucal e geral em bons estados, qual a importância de uma boa saúde bucal para um paciente diabético, entre outras. Além dessas questões, os pesquisadores coletaram o valor da glicemia capilar da ficha dos pacientes. Os dados foram analisados e apresentados por meio de medidas de tendência central e de dispersão e ainda foi feito o teste estatístico qui-quadrado.

A Associação Americana de Diabetes ${ }^{12}$ definiu valores de referência de glicemia capilar para diagnostico de Diabetes Mellitus (Tabela 1). Entretanto, os pacientes da feira de saúde universitária não estavam em jejum durante a realização do teste de glicemia capilar, não informaram se estavam sob uso de insulina ou outro medicamento e a intenção desta pesquisa não foi diagnosticar a doença nos pacientes. Dessa forma, classificou-se, neste trabalho, como hiperglicemia (primeira alteração do nível glicêmico) os valores de glicemia capilar acima de $140 \mathrm{mg} / \mathrm{dl}$, já que os pacientes não estavam em jejum e houve intenção de detectar quaisquer alterações do nível glicêmico, não necessariamente Diabetes mellitus.

Vale ressaltar que no dia da entrevista não foi possível colher dados da glicemia em jejum dos pacientes e nem ter acesso ao prontuário médico para saber se são diagnosticados com Diabetes Mellitus e nem se estão em uso de insulina ou algum medicamento oral que diminua a glicemia. Portanto, o cálculo para prevalência de pacientes hiperglicêmicos em atenção multiprofissional pode ter sido prejudicado.

O trabalho atende aos quesitos primordiais da ética em pesquisa (Conselho Nacional De Saúde, 1996). Todos os pacientes assinaram o termo de consentimento livre e esclarecido. O projeto de pesquisa foi submetido à análise do Comitê de Ética em Pesquisa Envolvendo Seres Humanos da Universidade de Mogi das Cruzes, e aprovado sob o parecer $n^{\circ} 1.696 .531$ em agosto de 2016 . Esta pesquisa não teve financiamento e nem envolveu conflito de interesses.

Tabela 1- Valores de glicose plasmática para diagnóstico de Diabetes Mellitus e seus estágios pré-clínicos

\begin{tabular}{lccc}
\hline Categorias & $\begin{array}{c}\text { Jejum } \\
\text { (Mínimo de } 8 \text { horas) }\end{array}$ & $\begin{array}{c}\text { 2h após } \\
\mathbf{7 5 g} \text { de glicose }\end{array}$ & Glicemia ao acaso \\
\hline Normal & $<100 \mathrm{mg} / \mathrm{dl}$ & $<140 \mathrm{mg} / \mathrm{dl}$ & - \\
\hline Pré Diabetes & $100-125 \mathrm{mg} / \mathrm{dl}$ & $140-199 \mathrm{mg} / \mathrm{dl}$ & - \\
\hline Diabetes Mellitus & $>=126 \mathrm{mg} / \mathrm{dl}$ & $>=200 \mathrm{mg} / \mathrm{dl}$ & $>=200 \mathrm{com}$ polidipsia, perda inexplicável de peso \\
\hline
\end{tabular}

Fonte: Associação Americana de Diabetes (2017)

\section{RESULTADOS}

Dos 196 pacientes da Feira de Saúde 2016 no município de Mogi das Cruzes, 65 deles (33,1\%) eram do sexo masculino e $131(66,9 \%)$ do sexo feminino. A faixa etária geral variou de 18 a 77 anos, dando uma média de idade de 42 anos (DP \pm 7,07 anos).

Sobre os 65 homens da amostra, a média de idade desse grupo foi 43,4 anos e a de glicemia capilar foi 105,5 $\mathrm{mg} / \mathrm{dl}$, sendo que 6 deles estavam hiperglicêmicos. Já o grupo das 131 mulheres, a média de idade foi 41,3 anos e de glicemia foi 100,6 mg/dl, sendo que 9 delas estavam com hiperglicemia. Com isso, notou-se que a prevalência de hiperglicêmicos na amostra foi 7,7\%, sendo de $9,2 \%$ no sexo masculino e $6,9 \%$ no sexo feminino, sem diferença estatisticamente significativa $(p=0,51)$. Entre os pacientes considerados hiperglicêmicos, a média de glicemia foi de 226,2 mg/dl (DP $\pm 72,25)$.
No questionário aplicado havia perguntas para avaliar se o dentista e o médico dos pacientes entrevistados os orientavam sobre a necessidade de ter uma boa saúde bucal para auxiliar no controle glicêmico. A amostra foi dividida em quatro grandes grupos de acordo com o tipo de orientação que recebiam: apenas pelo seu médico, apenas pelo seu dentista, nenhuma orientação ou orientação em conjunto por ambos os profissionais. O Gráfico 1 mostra que a minoria dos pacientes recebe instruções simultâneas da equipe multidisciplinar a respeito da necessidade do cuidado integral para melhor sucesso no controle glicêmico. Uma parte significativa da amostra (37\%) não recebeu nenhum tipo de informação sobre a influência da saúde bucal no controle glicêmico, nem por médico e nem por dentistas. 
Bilitardo IO, et al. A influência da saúde bucal no controle glicêmico, sob orientação interdisciplinar

Gráfico 1 - Comparação entre o número de paciente e o tipo de orientação dada a eles na Feira de Saúde em Mogi das Cruzes (SP), 2016

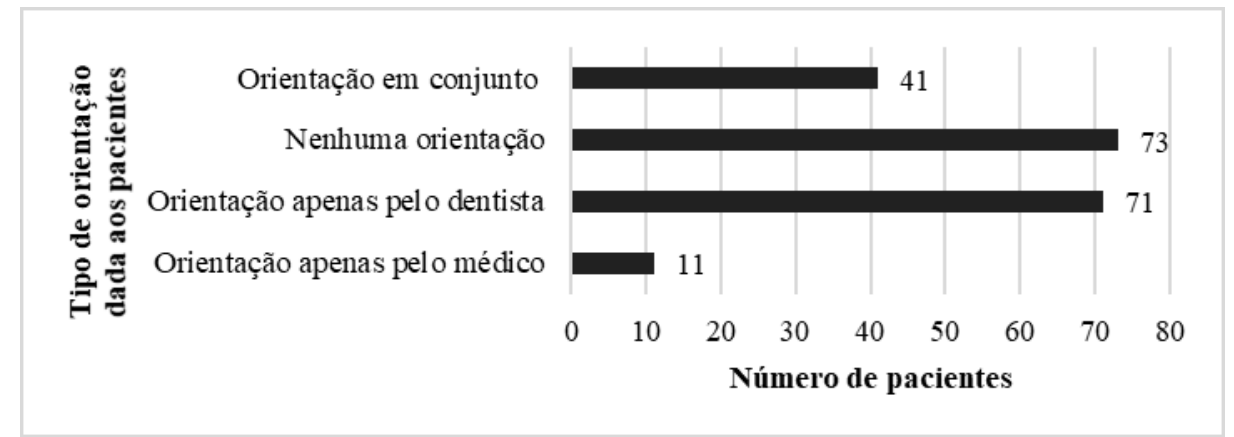

Fonte: elaboração própria

No Gráfico 2 nota-se que, o trabalho multidisciplinar e integral do dentista com o médico na orientação adequada do paciente, resulta, no geral, em menor média glicêmica (89.21 mg/dl), no grupo que nunca recebeu orientação, a média glicêmica foi de 105,9 mg/dl, a diferença entre a média dos dois grupos foi estatisticamente significativa $(\mathrm{p}=0,034)$. Entre àqueles que receberam orientação do

médico e dentista, ninguém foi classificado como tendo hiperglicemia, ou seja, todos possuíam nível glicêmico abaixo de $140 \mathrm{mg} / \mathrm{dl}$. Entretanto, no grupo que respondeu que ninguém nunca os informou sobre a importância da saúde bucal no controle glicêmico, seis foram classificados como hiperglicêmicos, e a média de glicemia entre esses foi de $220 \mathrm{mg} / \mathrm{dl}$.

Gráfico 2 - Comparação entre média de glicemia e tipo de orientação dada aos pacientes na Feira de Saúde em Mogi das Cruzes (SP), 2016

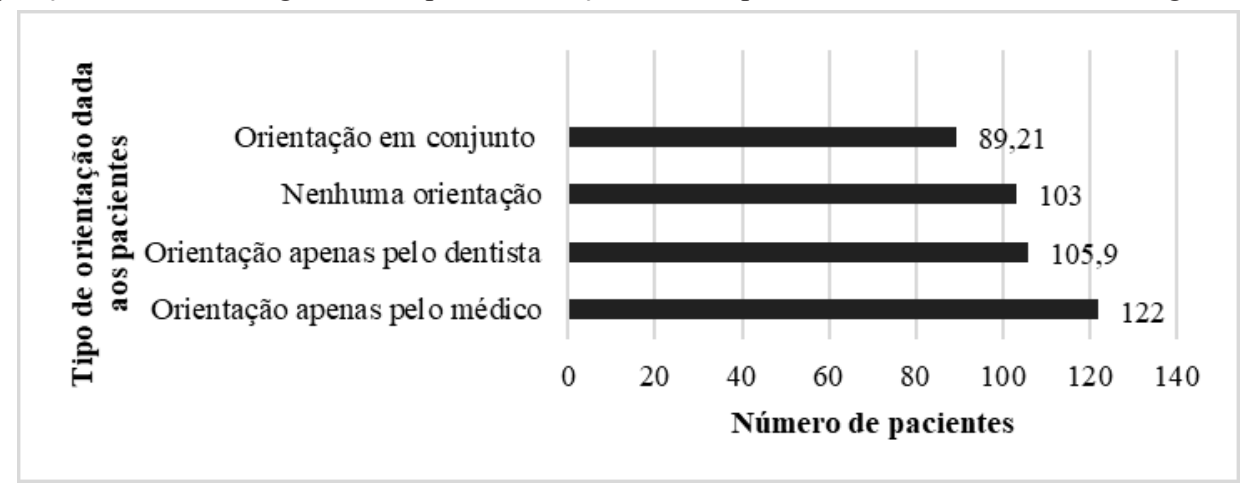

Fonte: elaboração própria

Ao analisar os dados sobre a condição da saúde bucal dos pacientes da amostra, notou-se que 132 deles $(67,3 \%)$ fazem uso de fio dental. Dentro desse grupo que passa fio dental frequentemente, dos 50 que possuem sangramento gengival durante o processo, 4 estavam hiperglicêmicos (8\%), enquanto dos 82 que não apresentam sangramento com o uso do fio dental, 6 estavam hiperglicêmicos (7,3\%).

Em toda a amostra, 69 pacientes $(35,2 \%)$ afirmaram que têm sangramento gengival, sendo que 15 pacientes (22\%) desse grupo são amplamente orientados pelo médico e dentista sobre a relação de uma boa saúde bucal e geral, ao ponto que 34 pacientes (49\%) recebem orientação de apenas um tipo de profissional de saúde e 20 pacientes (29\%) não recebem orientação nenhuma quanto a essa informação. $\mathrm{Na}$ análise estatística dos dados da Tabela 2, na qual se relacionou sangramento gengival e educação dos pacientes sobre a influência de uma boa saúde bucal na saúde geral, por médicos e dentistas em conjunto, obteve-se $\mathrm{RR}=1,05$ $\left(\mathrm{X}^{2}=0,04 ; \mathrm{p}<0,9\right)$.

Tabela 2- Relação entre sangramento gengival e educação dos pacientes por meio da equipe médica e odontológica em conjunto

\begin{tabular}{|c|c|c|c|c|c|c|c|}
\hline & & \multirow{2}{*}{\multicolumn{2}{|c|}{$\begin{array}{c}\text { Sangramento } \\
\text { Sim }\end{array}$}} & \multirow{2}{*}{\multicolumn{2}{|c|}{$\begin{array}{c}\text { Gengival } \\
\text { Não }\end{array}$}} & \multirow{2}{*}{\multicolumn{2}{|c|}{$X^{2}=0,04$}} \\
\hline & & & & & & & \\
\hline & & $\mathbf{n}$ & $\%$ & $\mathbf{n}$ & $\%$ & Total & $p<0,9$ \\
\hline \multirow{2}{*}{$\begin{array}{l}\text { Educação por meio de médicos e } \\
\text { dentistas em conjunto sobre uma } \\
\text { boa saúde bucal influenciar na } \\
\text { saúde geral }\end{array}$} & Sim & 15 & 7,65 & 26 & 13,26 & \multicolumn{2}{|c|}{41} \\
\hline & Não & 54 & 27,55 & 101 & 51,53 & \multicolumn{2}{|c|}{155} \\
\hline Total & & 69 & 35,2 & 127 & 64,8 & \multicolumn{2}{|c|}{196} \\
\hline
\end{tabular}


Ainda sobre os 69 pacientes que possuem sangramento gengival, ou seja, apresentam uma gengiva doente, 36 pacientes (52\%) acreditam ter uma saúde bucal boa ou muito boa.

Em relação à avaliação do conhecimento dos pacientes desta pesquisa sobre a integração da saúde bucal com a saúde geral nesse estudo, 177 entrevistados (90,3\%) acreditam que a integração do acompanhamento médico e odontológico é importante ou muito importante. Entretanto, apenas 76 entrevistados $(38,7 \%)$ consideram que a saúde bucal é importante especificamente para auxiliar a controlar a glicemia capilar de um paciente com Diabetes Mellitus. Dos pacientes que têm essa opinião, $62 \%$ receberam pelo menos orientação de um profissional de saúde sobre a questão de a saúde ser tratada como um todo e $65 \%$ afirmaram que não possuem sangramento gengival.

Agora, apenas sobre os 15 pacientes hiperglicêmicos da amostra, 7 (46,6\%) deles possuem sangramento gengival, nenhum recebe orientação das equipes médica e odontológica em conjunto, 9 (60\%) recebe orientação de uma equipe profissional de saúde, seja ela médica ou odontológica, e 6 (40\%) não recebe orientação de nenhuma equipe profissional de saúde. É importante ressaltar que $4(26 \%)$ pacientes deste grupo relataram que nunca vão ao médico responsável pela hiperglicemia e outros 3 (20\%) pacientes nunca vão ao dentista. Apenas $4(26,6 \%)$ pacientes acreditam que uma boa saúde bucal pode afetar no controle da glicemia capilar, e desses, 3 pacientes receberam esta informação durante consultas médicas ou odontológicas.

\section{DISCUSSÃO}

A partir do século XX, notou-se uma fragmentação do trabalho médico. Como consequência disso, as especializações reduziram consideravelmente a atuação clínica abrangente de cada médico especialista. Isso fez com que os pacientes, ao longo dos anos, se acostumassem com consultas focadas em solicitação de exames e dispensação de medicamentos. Por isso, é comum perceber resistência na população quando há tentativas de instalar práticas de atendimento de saúde que fujam daquilo que ela já está adaptada ${ }^{13}$.

Um dos motivos para algumas unidades de atenção primária à saúde (UBS e ESF) não desenvolverem atividades de grupo para pessoas diabetes, por exemplo, é a baixa adesão da população resistente a essas novas propostas, talvez pela baixa escolaridade de grande parte dela ${ }^{14,15}$. Os motivos encontrados foram não só que a população em geral interessa-se mais pelas consultas médicas, o que aumenta a demanda nas unidades e impossibilita o desenvolvimento de ações educativas, mas também pelo fato dela desconhecer ainda os riscos inerentes às doenças e a importância desse tipo de estratégia ${ }^{13,16,17}$.

A falta de conhecimento dos pacientes diabéticos e de seus cuidadores, associada à inadequada capacitação e integração entre os profissionais de saúde, relacionase diretamente ao problema da adesão ao tratamento tradicional, fazendo diferença para a saúde e qualidade de vida do paciente. Compreende-se que a equipe multiprofissional de saúde precisa dialogar com os pacientes para fazê-los compreender o processo saúdedoença para melhor adesão às propostas da equipe, além da equipe dialogar mais entre si para realizar atendimentos baseados no princípio da integralidade proposto pelo SUS em $1988^{10}$.

Um estudo envolveu dez pacientes diabéticos e a maioria (sete) deles respondeu que seus cuidados com a saúde bucal são iguais aos da população em geral e não demonstraram saber que por serem diabéticos mal controlados possuem maior chance de adquirir doença periodontal. Além disso, informaram não receber qualquer tipo de orientação sobre a relação existente entre saúde bucal e Diabetes Mellitus ${ }^{18}$. De modo muito semelhante ao presente estudo, dentre os 15 pacientes da feira de saúde que foram enquadrados como hiperglicêmicos, $40 \%$ deles relataram não receber nenhuma informação a respeito dessa relação e, dentre aqueles que foram educados de algum modo sobre Diabetes Mellitus durante as consultas, nenhum relatou receber orientações por mais de um profissional de saúde. Mesmo esses pacientes possuírem glicemia elevada, apenas $26,6 \%$ acreditam que ela pode ser afetada por alterações na condição bucal de saúde, um número baixo. Entretanto, foi interessante notar que $3 / 4$ desses hiperglicêmicos que possuem consciência do cuidado não só geral como bucal sabem disso porque foram educados nas consultas que realizaram nos últimos tempos, mostrando que a educação ao paciente se faz necessária para que ele possa compreender seu processo de saúde doença e colaborar com as propostas de tratamento sugeridas pelas equipes.

Além da preocupação da saúde bucal influenciar o controle glicêmico, vale ressaltar a relação bidirecional entre elas, ou seja, havendo também consequências de um mal controle glicêmico na própria saúde bucal ${ }^{19}$. Exemplificando tal cenário, estudos reportaram a associação entre menor incidência de cárie e diabetes mellitus compensado, tendo a justificado pelo controle metabólico do diabetes, da dieta com baixo conteúdo de carboidratos, com o aumento de proteínas e aumento na capacidade tampão da saliva ${ }^{20}$. Outro estudo, com crianças entre 8 e 16 anos já diagnosticadas com Diabetes Tipo 1, foi visto que a maioria dos pacientes com níveis de hemoglobina glicada $>8 \%$ eram do sexo feminino e desenvolveram mais cárie dentária em dentes permanentes em relação aos pacientes com hemoglobina glicada $<8 \%$, devido a saliva possuir menor fluxo, maior concentração de glicose e favorecer a instalação da cárie ${ }^{21}$. Comparando esses outros estudos com o atual, apesar de o presente estudo ter apresentado a minoria de sua amostra com pacientes adultos hiperglicêmicos (15 pacientes - 
7,6\%), e sendo eles do sexo masculino predominantemente, continua sendo importante e válida a preocupação com o reflexo dessa glicemia na saúde bucal, pois além de cáries, pode comumente haver doença periodontal (gengivite e periodontite), independentemente de sexo e idade dos pacientes. Uma vez instalada a doença periodontal, forma-se um processo inflamatório crônico, o que piora a resistência à insulina, tanto em Diabetes tipo 1 quanto no Diabetes tipo 2.

Analisando o Gráfico 1, percebe-se que, na amostra geral, 73 pacientes $(37,2 \%)$ não recebem orientações nem por parte da equipe médica e nem pela odontológica sobre a importância de uma boa saúde geral em conjunto com boa saúde bucal para melhor controle da glicemia capilar, o que mostra a integração entre médicos e dentistas incipientes quanto à integralidade proposta pelo SUS para a maioria dos casos da amostra. Entretanto, se comparar os pacientes que recebem algum tipo de orientação sobre integralidade durante o atendimento de saúde, de modo geral, foi visto que os dentistas informam mais seus pacientes a respeito da conexão saúde bucal- saúde geral já que 112 (57,1\%) tiveram orientação em algum momento pelo dentista, contra apenas $52(26,5 \%)$ pelo médico.

Para a maioria desses pacientes, nota-se que teve uma atenção multidisciplinar, porém faltou uma atenção interdisciplinar, ou seja, uma intersecção das diferentes áreas da saúde em um mesmo momento de consulta. De acordo com o Gráfico 2, quando o médico e o dentista realizaram esse tipo de atendimento, a média glicêmica dos pacientes em números absolutos apresentou-se estatisticamente beneficiada $(\mathrm{p}=0,034)$, uma vez que estava em nível mais baixo $(89,21 \mathrm{mg} / \mathrm{dl})$ se comparada principalmente com a do grupo com orientação comprometida por ambas as equipes de saúde. A glicemia do grupo bem orientado pode ter tido o bom resultado por diversos motivos, sendo que estes não podem ser descartados: diferentes abordagens profissionais em um mesmo paciente, melhor diálogo entre equipe de saúde e paciente, maior compreensão e esclarecimento dos processos saúde-doença e melhor adesão a tratamentos indicados.

Apesar da informação odontológica técnica ter avançado cientificamente ao longo dos anos, ainda não se consegue uma comunicação efetiva com o saber popular. Em uma pesquisa com amostra de 40 indivíduos, 35\% deles tiveram sangramento na gengiva como principal sinal de periodontite, número que aparece no presente estudo de forma semelhante $(35,2 \%)$. Porém, discutiu-se que os pacientes só se preocuparam com a situação e procuraram um atendimento quando houve um amolecimento dos dentes $^{22}$. Por mais que o dentista oriente, muitas vezes o paciente não assimila da forma que deveria e acaba adiando a cura por não reconhecer os sinais de um problema bucal. Também não identificam que um tratamento que aparentemente seria só dentário beneficiaria a saúde como um todo.
No presente estudo, quando se analisou o grupo com sangramento na gengiva, notou-se que a minoria $(21,7 \%)$ era educada pela equipe médica e odontológica em conjunto, porém não houve significado estatístico ao comparar a presença de sangramento gengival com falta de educação recebida por profissionais da saúde durante consultas, já que $R R=1,05$ e $p<0,9$. Ainda assim vale ressaltar que uma maioria (52\%) que acredita ter uma saúde bucal boa ou muito boa, mesmo tendo relatado essa condição gengival patológica, evidenciando que para essa situação realmente ainda não se conseguiu uma comunicação efetiva com o saber popular.

Em contrapartida, apesar de apenas $38,7 \%$ acreditarem que a saúde bucal é mais importante para um paciente diabético, pois pode melhorar sua glicemia capilar, a maioria deles $(62 \%)$ disse ter sido orientada por ambas as equipes sobre a relação saúde bucal-saúde geral, mostrando que a educação fornecida durante os atendimentos impactou no conhecimento deles. Além disso, ver que quase todos os pacientes $(90,3 \%)$ citaram que consideram importante ou até mesmo muito importante o acompanhamento médico e o odontológico integrados, já pode ser um sinal de mudança no pensamento de atendimento fragmentado proposto no século $\mathrm{XX}$, como discutido anteriormente.

A competência de prevenção de agravos e de promoção de saúde têm algumas atribuições que são bem referidas para os grupos de trabalhadores tanto SUS e quanto não SUS. Elas envolvem participações em reuniões de equipe, contribuição no plano de ações da equipe e interação com outros setores em ações de promoção de saúde. Essas atribuições, quando bem aplicadas, refletirão diretamente na efetiva integralidade discutida até então $0^{23}$. Além disso, posturas profissionais diferenciadas, como escuta atenta, recepção humanizada do usuário com respostas adequadas, responsabilizando-se, gerando vínculo, humanização, cuidado e acolhimento, podem contribuir também ${ }^{24}$. Quando se trata de pacientes da faixa etária pediátrica, há evidência de que a aproximação da equipe com escolas do bairro, conseguem fortalecer o vínculo deles com a equipe e trazer essas crianças e jovens às unidades de saúde com maior facilidade, o que facilitaria adesão a medidas de intervenção, prevenção e promoção de saúde quando crescerem ${ }^{25}$.

Sabe-se que processos de municipalização e descentralização do SUS ampliaram os serviços públicos odontológicos com a preocupação de estabelecer o atendimento integral e multidisciplinar ao paciente. No entanto, apesar dos dados mostrarem que o dentista orientou mais seus pacientes de modo interdisciplinar que o médico, esses novos vínculos empregatícios da equipe odontológica muitas vezes levam a uma rotatividade grande de trabalhadores, podendo resultar em comprometimento do vínculo e integralidade com os pacientes e outros profissionais das unidades de saúde que atuam ${ }^{23,24}$.

Há outros fatores, além da falta de diálogo e 
interação entre as equipes, que podem influenciar o atendimento interdisciplinar preconizado, como a falta de inserção de conteúdos interdisciplinares nas redes de ensino superior para os cursos da área da saúde, principalmente medicina. Mostraram em estudos que o curso de medicina aborda poucos conteúdos odontológicos durante a graduação, já que apenas $29,7 \%$ dos médicos pediatras analisados receberam instruções sobre saúde bucal durante a graduação ${ }^{26}$.

Os métodos das instituições de ensino superior têm um papel primordial na formação desses profissionais, pois utilizar predominantemente métodos verticalizados, negligenciando a interação que deve haver entre os profissionais e os usuários de saúde, influenciará o modo que essas pessoas promoverão saúde futuramente ${ }^{27}$.

Como a integração ensino-serviço é vista como potencial colaboradora do processo de mudança de práticas na formação em saúde, a reestruturação curricular dos cursos de saúde, como inserir conteúdos interdisciplinares na grade, discutir importância disso na saúde geral do paciente e inserir os alunos em unidades de atenção primária à saúde é necessária para que eles compreendam esse processo na prática dos atendimentos. Como as UBSs vem se mostrando um cenário fértil para a aprendizagem, deixar o aluno vivenciar a experiencia de profissionais já inseridos nos princípios de integralidade faz a diferença ${ }^{28}$. A colaboração estreita entre o paciente, os profissionais de saúde primários e os profissionais de saúde oral pode ser uma alternativa de melhorar tanto a saúde geral quanto a bucal do paciente diabético, já que indivíduos com diabetes tipo 2 em algumas condições orais apresentaram pior saúde geral ${ }^{29}$.

\section{CONCLUSÃO}

Dar qualidade à saúde bucal de um diabético não cura a doença, porém o auxilia no controle glicêmico de alguma forma, caso ele tenha periodontite ou infecção bucal grave. Salienta-se a necessidade não só de o estudante de medicina saber da influência da saúde bucal na saúde geral, mas também de ter serviços de saúde públicos e privados focados na educação dos pacientes, oferecendo atendimento multiprofissional integral a eles, já que a minoria conhece a relação entre a saúde bucal com a saúde geral. Assim, haverá promoção do autocuidado, comunicação entre as diversas áreas da saúde e melhor cuidado desses pacientes, diminuindo as complicações causadas pela hiperglicemia, as quais afetam a qualidade de vida dessa relevante parcela da população.

Agradecimentos: Agradecemos à Organização Mogiana de Educação e Cultura (OMEC) e ao Centro Acadêmico Antonio Prudente (C.AA.P.) por meio do XVI Projeto Antonio Prudente - Feira de Saúde, que colaboraram com a realização deste artigo.

Participação dos autores: Os autores desta publicação contribuíram de modo equilibrado sob os seguintes aspectos principais: Bilitardo $I O$ - participou na criação das ideias iniciais deste artigo. Apoiou desde o planejamento da pesquisa e a coleta de dados em campo, bem como as análises de dados e a redação do artigo. Leite $B N$ - participou na criação das ideias iniciais deste artigo. Apoiou desde o planejamento da pesquisa e a coleta de dados em campo, bem como as análises de dados e a redação do artigo. Mello TRC - orientadora do trabalho, apoiou desde o início o desenvolvimento dele. Orientou o planejamento da pesquisa e a coleta de dados em campo, bem como auxiliou nas análises de dados e na redação do artigo.

\section{REFERÊNCIAS}

1. Câmara SAV, Barbosa TS, Olivan VC, Fernandes ALP, Câmara JV. Avaliação do risco para desenvolvimento de diabetes mellitus tipo 2 em estudantes universitários. Rev Ciên Plural (Natal). 2019;5(2):94-110. https://doi. org/10.21680/2446-7286.2019v5n2ID18129.

2. Iser BPM. Prevalência de diabetes autorreferida no Brasil: situação atual e tendências [doutorado]. Rio Grande do Sul: Universidade Federal do Rio Grande do Sul; 2016. Disponível em: ttp://hdl.handle.net/10183/149588.

3. De Souza Porcelli IC, Corsi NM, Barata TJE, Moura SK, Cardelli AAM, Poli-Frederico RC, Macielet SM. Comparação do padrão de saúde bucal entre idosos diabéticos e não diabéticos de um município do sul do Brasil. Rev Uningá (Maringá). 2018;55(S3):115-27. Disponível em: http://revista.uninga.br/index.php/uninga/ article/view/2346.

4. Alves C, Andion J, Brandao M, Menezes R. Mecanismos patogênicos da doença periodontal associada ao diabetes melito. Arq Bras Endocrinol Metab (São Paulo).
2007;51(7):1050-7. https://doi.org/10.1590/S000427302007000700005.

5. Silva Jr GF, Lessa EF, Mendes AS. Avaliação do risco sistêmico para diabetes mellitus e doença cardíaca coronariana em pacientes portadores de periodontite. Rev JOPIC UNIFESO (Teresópolis). 2016;1(1):9-23. Disponível em http://unifeso.edu.br/revista/index.php/jopic/article/ view/189.

6. Sousa JNL, Nóbrega DRDM, Araki ÂT. Perfil e percepção de diabéticos sobre a relação entre diabetes e doença periodontal. Rev Odontol UNESP (Araraquara). 2014;43(4):265-72. https://doi.org/10.1590/rou.2014.042

7. De Aguiar Gonçalves JL, Ribeiro EOA, Prestes GR, Soares KS. Avaliação da condição bucal de pacientes com doença renal crônica em tratamento na Fundação Hospital Adriano Jorge - AM. Arq Odontol. 2019;55:18. Disponível em: https://periodicos.ufmg.br/index.php/ arquivosemodontologia/article/view/12715.

8. Pavesi LA. Alterações nos parâmetros clínicos médicos após 
o tratamento da periodontite nos pacientes em hemodiálise [dissertação]. São Paulo: Universidade de Santo Amaro; 2016. Disponível em: http://hdl.handle.net/123456789/143.

9. Simpson TC, Needleman I, Wild SH, Moles DR, Mills EJ. Treatment of periodontal disease for glycaemic control in people with diabetes. Australian Dental J. 2010;55(4):4724. https://doi.org/10.1111/j.1834-7819.2010.01273.x.

10. Costa JA, Balga RSM, Alfenas RCG, Cotta RMM. Promoção da saúde e diabetes: discutindo a adesão e a motivação de indivíduos diabéticos participantes de programas de saúde. Ciênc Saúde Coletiva (Rio de Janeiro). 2011;16(3):2001-9. http://dx.doi.org/10.1590/S1413-81232011000300034

11. Instituto Brasileiro de Geografia e Estatística - IBGE. O que pesquisou? [citado 22 abr. 2018]. Disponível em: https://www.ibge.gov.br/estatisticas-novoportal/por-cidadeestado-estatisticas.html?t=destaques\&c=3530607. Link???

12. American Diabetes Association - ADA. Diagnosis [cited 2017 March 28]. Available from: http://www.diabetes.org/ diabetes-basics/diagnosis/?loc $=$ db-slabnav.

13. Mendonça FF, Nunes EFPA. Avaliação de grupos de educação em saúde para pessoas com doenças crônicas. Trab Educ Saúde (Rio de Janeiro). 2015;13(2):397-409. http://dx.doi.org/10.1590/1981-7746-sip00053.

14. Santos MA. Fatores que influenciam na baixa adesão ao tratamento da hipertensão arterial dos usuários da Unidade Básica de Saúde do Triângulo [Trabalho de conclusão de Curso de Especialização]. Formiga, MG: Universidade Federal de Minas Gerais; 2017. http://dx.doi. org/10.1590/1981-7746-sip00053.

15. Moutinho CB, Almeida ER, Leite MTS, Vieira MA. Dificuldades, desafios e superações sobre educação em saúde na visão de enfermeiros de saúde da família. Trab Educ Saúde (Rio de Janeiro). 2014;12(2):253-72. http:// dx.doi.org/10.1590/S1981-77462014000200003.

16. Melo G, Santos RM, Trezza MCSF. Entendimento e prática de ações educativas de profissionais do Programa Saúde da Família de São Sebastião-AL: detectando dificuldades. Rev Bras Enferm (Brasília). 2005;58(3):290-5. http://dx.doi. org/10.1590/S0034-71672005000300007.

17. Moura ERF, Sousa RA. Educação em saúde reprodutiva: proposta ou realidade do Programa Saúde da Família. Cad Saúde Pública (Rio de Janeiro). 2002;18(6):1809-11. http:// dx.doi.org/10.1590/S0102-311X2002000600038.

18. Gil GP, Haddad MCL, Guariente MHDM. Conhecimento sobre diabetes mellitus de paciente atendidos em programa ambulatorial interdisciplinar de um hospital universitário público. Semina: Ciênc Biol Saúde (Londrina). 2008;29(2):141-54. http://dx.doi.org/10.5433/16790367.2008v29n2p141.

19. Bailoordurgesh N, Hani AT, MohemadR N, Bassuoni MW, Al Shamrani A, Al-Thomali Y. The bi-directional relationship between diabetes mellitus and periodontal disease a structured review and contemporary concepts. IOSR J Dental Med Sci. 2017;16(5):81-6. doi: 10.9790/08531605098186.
20. Puttaswamy KA, Puttabudhi JH, Raju S. Correlation between salivary glucose and blood glucose and the implications of salivary factors on the oral health statusin type 2 diabetes mellitus patients. J Int Soc Prev Commun Dent. 2017;7(1):28-33. doi: http://dx.doi.org/10.4103/22310762.200703 .

21. Carneiro VL, Fraiz FC, Ferreira FM, Pintarelli TP, Oliveira $\mathrm{ACB}$, Boguszewski MCS. The influence of glycemic control on the oral health of children and adolescents with diabetes mellitus type 1. Arch Endocrinolol Metab (São Paulo). 2015;59(6):535-40. https://doi.org/10.1590/23593997000000117.

22. Nuto SAS, Nations MK, Costa ÍCC. Aspectos culturais na compreensão da periodontite crônica: um estudo qualitativo. Cad Saúde Pública (Rio de Janeiro). 2007,23(3):681-90. https://doi.org/10.1590/S0102-311X2007000300026.

23. Warmling CM, Rosa EK, Pezzato LM, Toassi RFC. Competências de auxiliares e técnicos de saúde bucal e o vínculo com o Sistema Único de Saúde. Trab Educ Saúde (Rio de Janeiro). 2016;14(2):575-92. https://doi. org/10.1590/1981-7746-sip00116.

24. Sanchez HF, Werneck MAF, Amaral JHL, Ferreira EF. A integralidade no cotidiano da atenção à saúde bucal: revisão de literatura. Trab Educ Saúde (Rio de Janeiro). 2015;13(1):201-14. https://doi.org/10.1590/1981-7746$\operatorname{sip} 00020$

25. Da Silva SLA, Barbosa LS, Aley NRL, Ribeiro TAV, Goyata SLT. Estratégia de Saúde da Família: dificuldade na atenção à saúde das crianças de 07 a 09 anos de idade. Rev Interdisciplinar Ciên Méd (Belo Horizonte). 2017;1(2):2635. Disponível em: http://revista.fcmmg.br/ojs/index.php/ ricm/article/view/19

26. Dalto V, Turini B, Cordoni Junior L. Conhecimento e atitudes de pediatras em relação à cárie dentária. Interface (Botucatu). 2008;12(24):205-10. https://doi.org/10.1590/ S1414-32832008000100016.

27. Gazzinelli MFC, Marques RC, Oliveira DC, Amorim MMA, Araújo EG. Representações sociais da educação em saúde pelos profissionais da equipe de saúde da família. Trab Educ Saúde (Rio de Janeiro). 2013;11(3):553-71. https:// doi.org/10.1590/S1981-77462013000300006.

28. Carvalho SOB, Duarte LR, Guerreiro JMA. Parceria ensino e serviço em unidade básica de saúde como cenário de ensino-aprendizagem. Trab Educ Saúde (Rio de Janeiro). 2015;13(1):123-44. https://doi.org/10.1590/1981-7746sip00026.

29. Sandberg GE, Sundberg HE, Fjekkstrim CA, Wikblad KF. Type 2 diabetes and oral health: a comparison between diabetic and non-diabetic subjects. Diabetes Res Clin Pract (Oxford). 2000;50(1):27-34. Disponível em: https://doi. org/10.1016/S0168-8227(00)00159-5.

Recebido: 03/07/2018

Aceito: 14/05/2020 